ARTICLE

\title{
Coherent transport through a Majorana island in an Aharonov-Bohm interferometer
}

\author{
A. M. Whiticar (1) 1,7, A. Fornieri (1) 1,7, E. C. T. O'Farrell', A. C. C. Drachmann (1) 1, T. Wang 2,3, C. Thomas ${ }^{2,3}$,

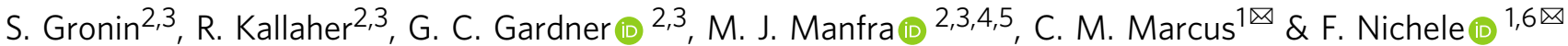

Majorana zero modes are leading candidates for topological quantum computation due to non-local qubit encoding and non-abelian exchange statistics. Spatially separated Majorana modes are expected to allow phase-coherent single-electron transport through a topological superconducting island via a mechanism referred to as teleportation. Here we experimentally investigate such a system by patterning an elongated epitaxial InAs-Al island embedded in an Aharonov-Bohm interferometer. With increasing parallel magnetic field, a discrete sub-gap state in the island is lowered to zero energy yielding persistent 1e-periodic Coulomb blockade conductance peaks ( $e$ is the elementary charge). In this condition, conductance through the interferometer is observed to oscillate in a perpendicular magnetic field with a flux period of $h / e$ ( $h$ is Planck's constant), indicating coherent transport of single electrons through the islands, a signature of electron teleportation via Majorana modes.

\footnotetext{
${ }^{1}$ Center for Quantum Devices, Niels Bohr Institute, University of Copenhagen and Microsoft Quantum Lab Copenhagen, Universitetsparken 5, Copenhagen 2100, Denmark. ${ }^{2}$ Department of Physics and Astronomy and Microsoft Quantum Lab Purdue, Purdue University, West Lafayette, IN 47907, USA. ${ }^{3}$ Birck Nanotechnology Center, Purdue University, West Lafayette, IN 47907, USA. ${ }^{4}$ School of Materials Engineering, Purdue University, West Lafayette, IN 47907 , USA. ${ }^{5}$ School of Electrical and Computer Engineering, Purdue University, West Lafayette, IN 47907, USA. ${ }^{6}$ Present address: IBM Research - Zurich,

Sumerstrasse 4, 8803 Rschlikon, Switzerland. 7 These authors contributed equally: A. M. Whiticar, A. Fornieri. ${ }^{凶}$ email: marcus@nbi.ku.dk; fni@ibm.zurich.com
} 
nitial experiments reporting signatures of Majorana zero modes (MZMs) in hybrid superconductor-semiconductor nanowires focussed on zero-bias conductance peaks (ZBPs) using local tunneling spectroscopy ${ }^{1-4}$. Subsequently, Majorana islands provided additional evidence of MZMs based on nearly $1 e$-spaced Coulomb blockade (CB) peaks ${ }^{5}$, and indicated a Rashba-like spin-orbit coupling with the spin-orbit field lying inplane, perpendicular to the wire axis ${ }^{6}$. Under some circumstances, these signatures can be mimicked by trivial modes ${ }^{7-9}$, motivating a new generation of experiments that explicitly probe non-local properties, which are more difficult to mimic. For instance, non-locality of MZMs was recently investigated by measuring the energy splitting induced by the interaction of a quantum dot and a zero-energy state in a hybrid nanowire ${ }^{10}$.

Non-locality can also be accessed by interferometric measurements of a Majorana island, where CB couples separated MZMs and fixes fermion parity ${ }^{1-15}$. In the topological regime, a Majorana island can coherently transfer a single-electron between its two ends through MZMs ${ }^{11,12}$. To demonstrate the effect, a Majorana island can be embedded in the arm of an Aharonov-Bohm (AB) interferometer. If single-electron transport in both the reference arm and the Majorana island is coherent, conductance through the interferometer is expected to show oscillations with a flux period $h / e^{11,16}$. In addition, interferometry offers a way to distinguish between localized trivial modes and $\mathrm{MZMs}^{12,14}$. This technique was used to investigate coherent transport in semiconductor quantum dots ${ }^{17-20}$.

In this work, we study coherent single-electron transport through a Majorana island with an $\mathrm{AB}$ interferometer. At high magnetic fields, we observe $1 e$ periodic $\mathrm{CB}$ peaks due to a discrete zero-energy state. In this case, we observe the conductance through the interferometer to oscillate periodically for a flux $h / e$ piercing the $\mathrm{AB}$ ring, while oscillations are suppressed when the island shows a $2 e$ periodicity or is in the normal state. The observation of conductance oscillations in the $1 e$ regime indicates coherent single-electron transport through the Majorana island, as predicted for electron teleportation mediated by $\mathrm{MZMs}^{11}$.

\section{Results}

Majorana island interferometer. Devices were fabricated using an InAs two-dimensional electron gas (2DEG) heterostructure covered by $8 \mathrm{~nm}$ of epitaxially grown $\mathrm{Al}^{21}$. The bare 2DEG (without $\mathrm{Al}$ ) showed a phase coherence length of $l_{\phi} \sim 4 \mu \mathrm{m}$ (see Supplementary Fig. 1). Figure 1a shows a micrograph of device 1 with a $1.2 \mu \mathrm{m}$ long and $0.1 \mu \mathrm{m}$ wide superconducting $\mathrm{Al}$ stripe formed by wet etching. Ti/Au top-gates were evaporated on top of a $25 \mathrm{~nm} \mathrm{HfO}_{2}$ dielectric grown by atomic layer deposition. We studied two lithographically similar interferometers with circumferences $L_{\text {loop }}$ of $5.6 \mu \mathrm{m}$ for device 1 and $5 \mu \mathrm{m}$ for device 2 .

Applying a negative voltage, $V_{\mathrm{pg}}$, to the central gate serves two purposes. It depletes the $2 \mathrm{DEG}$ surrounding the $\mathrm{Al}$ wire to form both the Majorana island and the $\mathrm{AB}$ ring center and also adjusts the chemical potential and charge occupancy of the island. Energizing all exterior gates confines the 2DEG into an $A B$ interferometer by connecting the Majorana island to a normal conducting reference arm. The resistance of the reference arm was independently tuned by a negative gate voltage $V_{c}$. A sourcedrain bias voltage $\left(V_{\mathrm{sd}}\right)$ was applied to one lead and the resulting current and four-terminal voltage was recorded. The in-plane magnetic fields $B_{\|}$and $B_{\mathrm{t}}$, and perpendicular field, $B_{\perp}$, were controlled by a three-axis vector magnet.

At low temperatures, tunneling of single electrons onto a Majorana island with a superconducting gap $\Delta$ is suppressed by $\mathrm{CB}$, except at charge degeneracies. When the lowest sub-gap state energy, $E_{0}$, exceeds the charging energy $E_{c}$, ground-state degeneracies only occur between even-occupied states, resulting in $2 e$-periodic CB conductance peaks ${ }^{22}$. Odd-occupied ground states are lowered into the accessible spectrum by a Zeeman field, resulting in even-odd $\mathrm{CB}$ peak spacing when $0<E_{0}<E_{\mathrm{c}}$. The difference in peak spacings between even and odd states, $S=S_{\mathrm{e}}-$ $S_{\mathrm{o}}$, is proportional to $E_{0}{ }^{5}$ (see Fig. $1 \mathrm{~b}$ ). For well-separated MZMs, $E_{0}$ tends exponentially toward zero, yielding $1 e$ periodic $\mathrm{CB}$ peaks with a discrete zero-bias state at consecutive charge degeneracy point ${ }^{5,23}$. Both observations are necessary for a MZM interpretation. When MZMs are not widely separated, CB peak spacings oscillate with field and chemical potential ${ }^{5-7}$.

CB spectroscopy. We first investigated the Majorana island without interferometry by depleting a segment of the reference arm (see Fig. 1a). Figure $1 \mathrm{~b}$ shows zero-bias differential conductance $G=\mathrm{d} I / \mathrm{d} V$ of the island as a function of parallel magnetic field $B_{\|}$and gate voltage $V_{\mathrm{pg}}$, which controls the electron occupancy and chemical potential of the island. CB peaks are $2 e$ periodic at zero field and split around $2 \mathrm{~T}$, becoming $1 e$ periodic as the sub-gap state moves toward zero energy (see Fig. 3a for peak spacing analysis). Performing $\mathrm{CB}$ spectroscopy, that is, measuring $G$ as a function of both source-drain bias $V_{\text {sd }}$ and $V_{\mathrm{pg}}$ reveals Coulomb diamonds (Fig. $1 \mathrm{c}-\mathrm{f}$ ). At low $B_{\|}$, diamonds are $2 e$ periodic with distinct negative differential conductance (Fig. 1c), which transition to an even-odd peak spacing difference at moderate fields (Fig. 1d), similar to previous work on superconducting Coulomb islands $5,6,22,24-27$. At high fields, the $1 e$ periodic diamonds show a discrete ZBP for consecutive charge degeneracy points that is well separated from the superconducting gap (Fig. 1e). This sub-gap feature remained at zero bias until the superconducting gap closure, and persists for $3 \mathrm{mV}$ in $V_{\mathrm{pg}}$, corresponding to an energy range of $0.8 \mathrm{meV}$. For systems with strong spin-orbit interaction, a helical gap of $g \mu_{\mathrm{B}} B_{\|}$is expected, where $\mu_{\mathrm{B}}$ and $g$ are the Bohr magneton and electron $g$-factor, respectively. We estimate a helical gap of $\sim 0.6 \mathrm{meV}$ at $B_{\|}=2.5 \mathrm{~T}$ for a $g$-factor of 4 , comparable to the span of the ZBP in $V_{\mathrm{pg}}$. The stability of the zero-bias state in both magnetic field and in chemical potential is consistent with the MZM picture (see Supplementary Fig. 2$)^{5}$, however, the observation of coherent single-electron transport is needed to draw conclusions about non-locality. Below, we additionally show that the zero-bias state is sensitive to rotations of the in-plane field. The magnitude of $B_{\|}$ where $1 e$ periodicity is observed is in agreement with ZBPs measured in tunneling spectroscopy in InAs $2 \mathrm{DEGs}^{3}$. In contrast, as a function of $B_{\mathrm{t}}$ the peak spacing showed an oscillating even-odd periodicity, and no consecutive ZBPs were observed (see Supplementary Fig. 3d-f), as expected for extended modes in the wire $e^{6,9,28}$. The normal state $1 e$ regime of the Majorana island appears above $B_{\|} \sim 3 \mathrm{~T}$ with $E_{\mathrm{c}}=80 \mu \mathrm{eV}$ (Fig. 1f), where no discrete ZBPs are observed (see Supplementary Figs. 2 and $3 \mathrm{a}-\mathrm{c}$ ).

Interferometry and coherent single-electron transport. The reference arm of the $\mathrm{AB}$ interferometer was connected to the Majorana island by tuning $V_{\mathrm{c}}$ from -8 to $-3 \mathrm{~V}$ while $V_{\mathrm{pg}}$ was compensated. This lifted the overall conductance by opening a path through the reference arm (see Fig. 2a-d). Fig. 2e-h shows the conductance $\Delta G$ through the full interferometer (with smooth background subtracted; see "Methods" section) as a function of $B_{\perp}$ and gate voltage $V_{\mathrm{pg}}$, which control the flux in the interferometer and occupancy of the island, respectively. Figure $2 \mathrm{e}$ shows small oscillations in $\Delta G\left(B_{\perp}\right)$ at $B_{\|}=0 \mathrm{~T}$ for the $2 e$ periodic peaks. For $B_{\|}=2.2 \mathrm{~T}$, where the peak spacing is even-odd (Fig. 2f), the conductance showed moderate oscillations with a period $\Delta B_{\perp}=1.5 \mathrm{mT}$. This periodicity corresponds to a single flux quantum $h / e$ threading an area of $\sim 2.7 \mu \mathrm{m}^{2}$, consistent with 
a

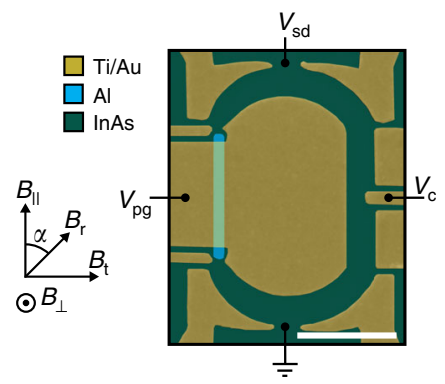

b

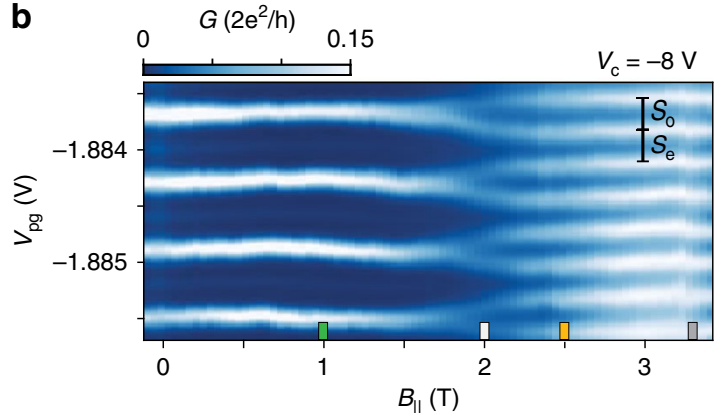

C

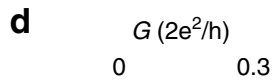

e $\quad G\left(2 e^{2} / h\right) \quad f$

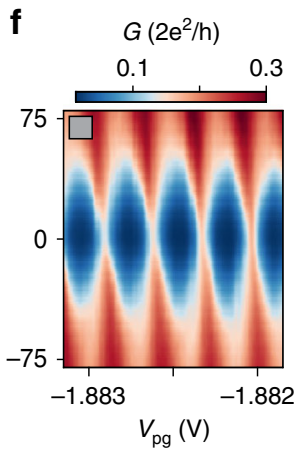

Fig. 1 Majorana island interferometer. a False-color electron micrograph of the Majorana island interferometer where an Al wire (light blue) is embedded in a normal conducting Aharonov-Bohm interferometer (green) defined by Ti/Au gates (yellow). The gate voltage $V_{p g}$ defines both the Majorana island and the interferometer center, and controls the electron occupancy. The gate voltage $V_{c}$ controls the resistance of the reference arm and $V_{s d}$ is the sourcedrain dc bias voltage. Magnetic field directions are shown with $\alpha$ denoting the in-plane angle measured with respect to the wire direction. Scale bar, $1 \mu \mathrm{m}$. b Zero-bias differential conductance $G$ as a function of $B_{\|}$and $V_{p g} . S_{e}\left(S_{0}\right)$ is the even (odd) CB peak spacing. c-f Differential conductance $G$ as a function of $V_{\mathrm{sd}}$ and $V_{\mathrm{pg}}$ showing Coulomb diamonds for $B_{\|}=1 \mathrm{~T} \mathbf{c}, 2 \mathrm{~T} \mathbf{d}, 2.5 \mathrm{~T} \mathbf{e}$, and $3.3 \mathrm{~T} \mathbf{f}$. The measurements shown in panels $\mathbf{b}-\mathbf{f}$ were taken with the reference arm closed.
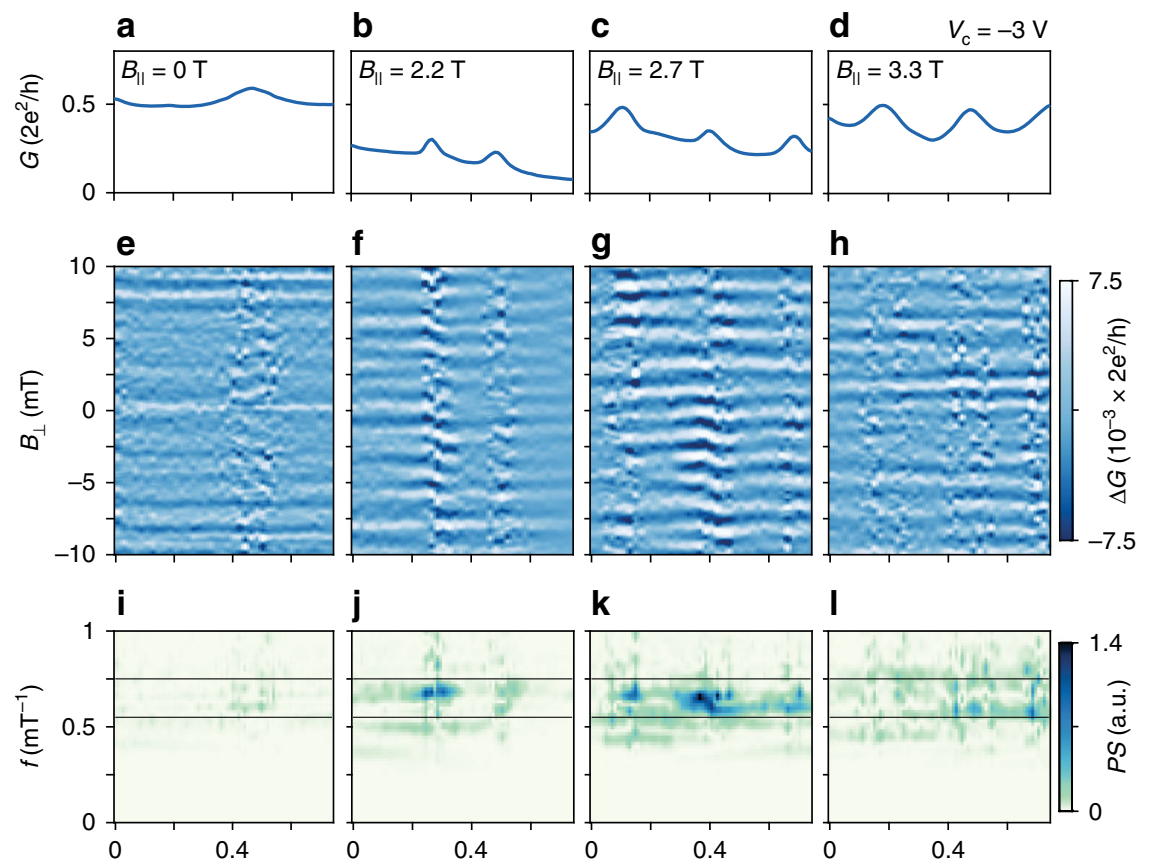

\section{k}
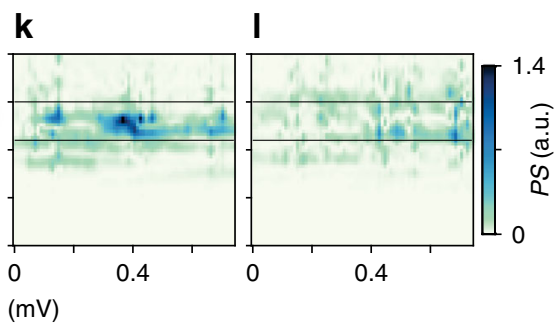

Fig. $2 \mathbf{h}$ /e periodic conductance oscillations. Magnetoconductance for parallel field values $B_{\|}=0,2.2,2.7$, and $3.3 \mathrm{~T}$ (left to right). a-d Zero-bias differential conductance $G\left(B_{\perp}=0\right)$ versus gate voltage $V_{\text {pg }}$ used to control electron occupation. e-h Conductance $\Delta G$ as a function of $V_{p g}$ and perpendicular magnetic field $B_{\perp}$ controlling the flux in the interferometer with corresponding power spectrums in i-l. The solid black lines indicate the frequency window bounding the Aharonov-Bohm oscillations (see "Methods" section). $\Delta G$ is the conductance with a subtracted slowly varying background. $\Delta V_{\mathrm{pg}}=0$ corresponds to $V_{\mathrm{pg}}=-1.896 \mathrm{~V}$. 

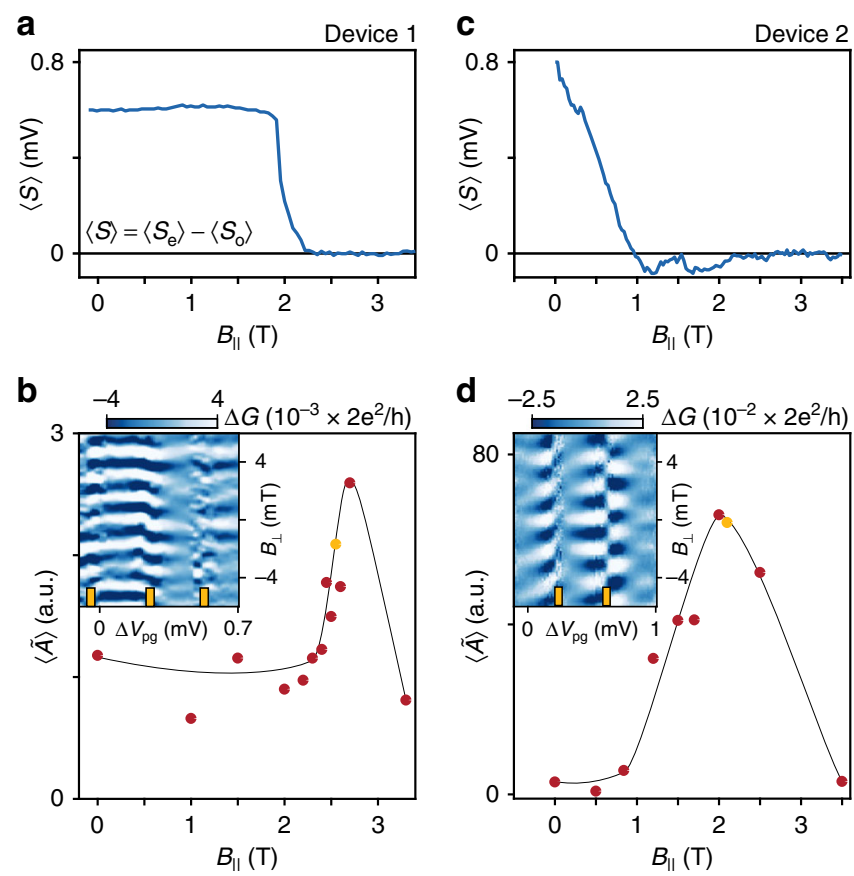

Fig. 3 Coherent single-electron transport. a, c Peak spacing difference $\langle S\rangle$ as a function of parallel magnetic field $B_{\|}$for devices 1 and 2. b, d AharonovBohm oscillation amplitude $\langle\tilde{A}\rangle$ as a function of $B_{\|}$. The solid line is a guide to the eye. Insets show characteristic magnetoconductance $\Delta G$ as a function of gate voltage $V_{\mathrm{pg}}$ controlling electron occupancy and perpendicular magnetic field $B_{\perp}$ controlling the magnetic flux in the interferometer in the 1e regime (indicated by yellow markers in the main panel). Yellow ticks show CB peak positions. $\Delta V_{\mathrm{pg}}=0$ corresponds to $V_{\mathrm{pg}}=-1.896$ and $-0.945 \mathrm{~V}$ for $\mathbf{b}$ and d, respectively.

the ring center area defined by $V_{\mathrm{pg}}$. This indicates coherent $1 e$ transport through both the reference arm and the Majorana island. At $B_{\|}=2.7 \mathrm{~T}$, the $\mathrm{CB}$ peak spacing is uniformly $1 e$, and oscillation amplitude is maximal (see Fig. 2g). When the Majorana island is driven normal, $B_{\|}>3 \mathrm{~T}$, conductance oscillations are reduced, becoming comparable to the low field oscillations (Fig. 2h). The appearance of strong h/e periodic conductance oscillations in the $1 e$ regime of the island is a key experimental signature of electron teleportation.

The strength and periodicity of the oscillations are examined more quantitatively using Fourier power spectrum (PS) analysis (see "Methods" section). In Fig. 2i-l, the PS of $\Delta G\left(B_{\perp}\right)$ are shown. Increasing $B_{\|}$leads to a peak appearing around $f=0.65 \mathrm{mT}^{-1}$, the frequency expected for $\mathrm{AB}$ interference. The PS is maximized in the $1 e$ regime. To quantify the oscillations amplitude, $\langle\tilde{A}\rangle$, we average the integrated PS (see "Methods" section).

We next correlate the $B_{\|}$dependence of the oscillations amplitude, $\langle\tilde{A}\rangle\left(B_{\|}\right)$, with the $B_{\|}$dependence of the lowest sub-gap state, $E_{0}\left(B_{\|}\right)$, of the island. The sub-gap energy is found from the difference between even and odd CB peak spacings, averaged separately, $\langle S\rangle=\left\langle S_{\mathrm{e}}\right\rangle-\left\langle S_{\mathrm{o}}\right\rangle$ (see Fig. 1b). In Fig. 3a, $\langle S\rangle$ remains constant as a function of $B_{\|}$(indicating $2 e$ transport) until a subgap state moves below $E_{c}$, reaching zero at $2.2 \mathrm{~T}$ without overshoot (as expected for well separated MZMs in a long wire $\left.\mathrm{e}^{5,23}\right)$. At low fields, where the $\mathrm{CB}$ periodicity is $2 e$, the oscillation amplitude $\langle\tilde{A}\rangle$ is small (Fig. 3b). When $\langle S\rangle$ approaches zero at high fields $\left(B_{\|}>2 \mathrm{~T}\right),\langle\tilde{A}\rangle$ exhibits a sharp increase that coincides with the $2 e$ to $1 e$ transition. Above $3 \mathrm{~T}$, the device is in the normal state and $\langle\tilde{A}\rangle$ returned to the low value found in the $2 e$ regime. This comparison shows that the oscillation amplitude is correlated with the energy of the lowest subgap state, and is maximal in the $1 e$ superconducting regime, as expected for electron teleportation.

Figure $3 c$, d shows a similar study for device 2. In Fig. $3 c,\langle S\rangle$ shows strong even-odd below $1 \mathrm{~T}$, fluctuates around $\langle S\rangle=0$ between 1 and $2 \mathrm{~T}$, then settles to $1 e(\langle S\rangle=0)$ above $2 \mathrm{~T}$. $\mathrm{CB}$ spectroscopy reveals a discrete state that oscillates around zero bias in both $B_{\|}$and $V_{\mathrm{pg}}$ without forming a stable $1 e$ periodic zerobias peak (see Supplementary Fig. 5). These oscillations about zero energy are compatible with hybridized Majorana modes due to wavefunction overlap resulting from the finite island length ${ }^{9,28}$. This overlap causes an energy splitting that oscillates both in field and chemical potential $5,6,27$. Figure $3 \mathrm{~d}$ shows that phase coherent transport first appears above $1 \mathrm{~T}$ and $\langle\tilde{A}\rangle$ gradually increases until reaching a maximum amplitude for $1 e$ peak spacing at $2.1 \mathrm{~T}$, before diminishing in the normal state. In comparison to device 1, phase coherent transport appears when $\langle S\rangle$ oscillations about zero, suggesting that hybridized Majorana modes characterized by an extended wavefunction may also contribute to coherent transport. The absence of energy oscillations may distinguish non-local MZMs from hybridized Majorana modes (see Fig. 3a, c). We attribute the reduced oscillation amplitude in device 1 to result from the longer loop length $L_{\text {loop }}>l_{\phi}$, leading to increased dephasing in the reference arm.

We observe that conductance oscillations measured on opposite sides of a $\mathrm{CB}$ peak in the $1 e$ regime are out of phase (see yellow ticks in the insets of Fig. 3) indicating a transmission phase shift of $\pi$ is acquired when the parity of the island is flipped. This demonstrates interferometric detection of island parity, which offers a way to detect MZM parity as proposed by several recent topological qubit schemes ${ }^{13,29,30}$. In some cases, we found that the the phase shift is restored through the $\mathrm{CB}$ valley, such that the same sides of adjacent $\mathrm{CB}$ peaks have the same phase. What determines whether there is phase recovery in the $\mathrm{CB}$ valley is not currently understood (see Supplementary Fig. 6) $15,18-20$.

The angular dependence of the in-plane magnetic field is investigated by fixing the field magnitude $B_{\mathrm{r}}=2.5 \mathrm{~T}$ and rotating the field by an angle $\alpha$ (see Fig. 1). Theoretically, a rotation of the in-plane field towards the Rashba field direction is expected to close the topological gap ${ }^{31}$. Figure $4 \mathrm{a}$ shows $1 e$ periodic Coulomb diamonds at $B_{\|}=2.5 \mathrm{~T}$ with a discrete $\mathrm{ZBP}$ at each charge degeneracy point (similar to Fig. 1e). Rotating by an angle $\alpha=5^{\circ}$ lifted the discrete state from zero energy, leading to even-odd peak spacing; at $\alpha=10^{\circ}$, 1e periodicity is recovered, though without a discrete ZBP. The observed sensitivity of the zeroenergy state to in-plane field rotation is consistent with $\mathrm{MZMs}^{31}$

Small rotations $\left(|\alpha|<7.5^{\circ}\right)$ reduced the oscillation amplitude, $\langle\tilde{A}\rangle$, as expected for even-odd periodicity (see Fig. 3 ). However, at larger angles $\left(|\alpha|>10^{\circ}\right)$ where the discrete ZBP is absent, a strong interference signal is observed (Fig. 4d). The observation of coherent transport in the absence of a discrete zero-energy state suggests trivial extended modes are also phase coherent over the length of the island. Therefore, the additional information provided by bias spectroscopy is needed to distinguish teleportation from other coherent transport mechanisms, as shown in Fig. $4 a-c$.

We further studied the effect of different magnetic field directions. The results are shown in Supplementary Fig. 7. In summary, all three axes showed coherent transport, with oscillation amplitude first increasing as $\langle S\rangle$ approached zero. This shows that the oscillation amplitude is dictated by the energy $E_{0}$ in all field directions and indicates that interference is not unique to a parallel magnetic field.

Finally, we comment on the physical mechanism that correlates the oscillation amplitude to the energy of $E_{0}$. At low 


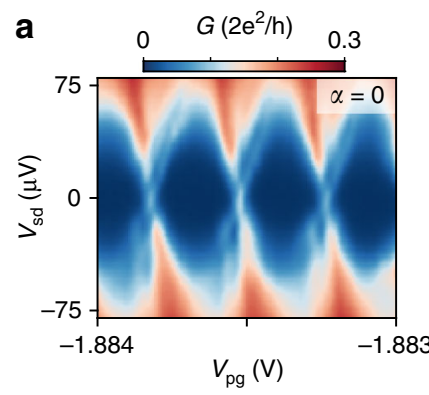

b
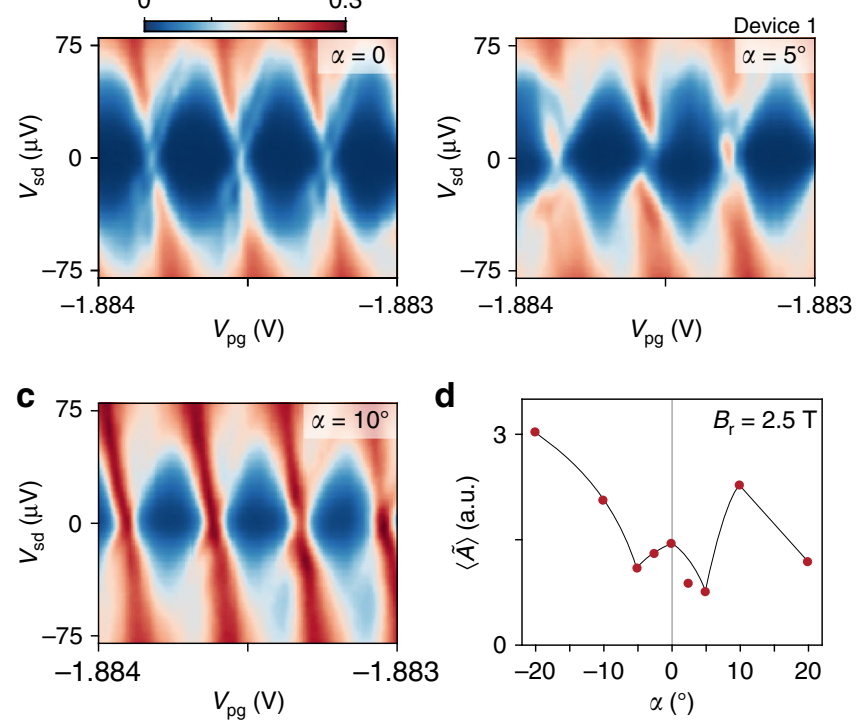

Fig. 4 In-plane magnetic field rotations. a-c Differential conductance $G$ as a function of gate voltage $V_{\text {pg }}$ controlling electron occupancy and source-drain bias voltage $V_{\text {sd }}$ showing Coulomb diamonds for in-plane rotation angles of $\alpha=0 \mathbf{a}, 5^{\circ} \mathbf{b}$, and $10^{\circ} \mathbf{c}$ with $\alpha=0$ corresponding to $B_{\|}=$ $2.5 \mathrm{~T}$. d Oscillation amplitude $\langle\tilde{A}\rangle$ as a function of in-plane rotation angle $\alpha$ for $B_{\mathrm{r}}=2.5 \mathrm{~T}$. The solid line is a guide to the eye.

fields, the Majorana island favors an even parity where transport of electrons occurs as two sequential tunneling events on either end of the island ${ }^{22,25}$. The two electrons acquire the condensate phase when forming a Cooper pair, which suppresses singleelectron coherence. At moderate fields, a discrete sub-gap state is brought below $E_{\mathrm{c}}$ and a single-electron transport channel is opened, allowing coherent resonant tunneling through the Majorana island. When the discrete state is brought to zeroenergy, the contribution of coherent transport is increased due to electron teleportation. Finally, in the normal state, we interpret the reduction in interference signal to reflect the short coherence length in the diffusive $\mathrm{Al}$ wire.

In conclusion, we report signatures of single-electron teleportation via non-local MZMs using $A B$ interference in combination with spectroscopy of a discrete zero-energy state. Our results also reveal that coherent transport by topologically trivial modes extending over the full length of the Majorana island are allowed. These extended trivial modes may be precursors of topological states in a finite length system that could transition into non-local MZMs by adjusting experimental parameters $^{28}$. We have shown that interferometry accompanying bias spectroscopy revealing stable $1 e$ periodic $\mathrm{CB}$ in magnetic field and chemical potential can discriminate non-local MZMs from extended modes (that display characteristic energy oscillations). Increasing the wire length to greatly exceed the diffusive coherence length $\xi=\sqrt{\xi_{0} l_{e}} \sim 1 \mu \mathrm{m}$ (for $\Delta=75 \mu \mathrm{eV}$ at $B_{\|}=$ $2.5 \mathrm{~T}$ ), where $\xi_{0}$ is the clean coherence length and $l_{\mathrm{e}} \sim 300 \mathrm{~nm}$ is the semiconducting mean free path will suppress $1 e$ transport via trivial extended modes ${ }^{32}$. The observation of coherent transport through the island rules out localized ABS at the ends of the wire as the source of the studied zero-bias state. Indeed, transport flips the parity of localized modes and suppresses interference, while transport through MZMs preserves island parity and coherent transport $^{12,14}$. These localized modes could have been a possible interpretation of the previously observed ZBPs in single-end tunneling experiments ${ }^{8,9}$.
These results suggest that InAs-Al 2DEGs are a promising route towards more complex experiments related to the braiding or fusion of MZMs. We have established coherent transport and parity readout from the transmission phase shifts in Majorana islands, two key results for future topological qubit networks ${ }^{13,29,30}$. Future devices will take advantage of improved material quality to allow for increased wire lengths to suppress coherent trivial quasiparticle transport, allowing MZM contributions to be better separated from other potential contributions.

\section{Methods}

Wafer structure. The devices were fabricated on wafers grown by molecular beam epitaxy on a InP substrate. The wafer stack consists of a $1 \mu \mathrm{m}$ graded $\operatorname{In}_{1-x} \mathrm{Al}_{x} \mathrm{As}$ insulating buffer, a $4 \mathrm{~nm} \mathrm{In} \operatorname{In}_{0.81} \mathrm{Ga}_{0.19}$ As bottom barrier, a $5 \mathrm{~nm}$ InAs quantum well, and a top barrier consisting of $5 \mathrm{~nm} \mathrm{In}_{0.9} \mathrm{Al}_{0.1}$ As for device 1 and $10 \mathrm{~nm}$ $\mathrm{In}_{0.81} \mathrm{Ga}_{0.19}$ As for device 2. A $7 \mathrm{~nm}$ film of epitaxial $\mathrm{Al}$ was then grown in situ without breaking the vacuum of the chamber. The InAs 2DEGs were characterized with a Hall bar geometry (Al removed), which showed a peak mobility of $\mu=$ $17,000 \mathrm{~cm}^{2} \mathrm{~V}^{-1} \mathrm{~s}^{-1}$ for an electron density of $n=1.7 \times 10^{12} \mathrm{~cm}^{-2}$ and $n=7.5 \times$ $10^{11} \mathrm{~cm}^{-2}$ for devices 1 and 2 , respectively.

Device fabrication. Devices were fabricated using standard electron beam lithography techniques. The devices were electrically isolated by etching mesa structures by first removing the top $\mathrm{Al}$ film with $\mathrm{Al}$ etchant Transene $\mathrm{D}$, followed by a deep III-V chemical wet etch $\mathrm{H}_{2} \mathrm{O}: \mathrm{C}_{6} \mathrm{H}_{8} \mathrm{O}_{7}: \mathrm{H}_{3} \mathrm{PO}_{4}: \mathrm{H}_{2} \mathrm{O}_{2}$ (220:55:3:3). Next, the Al film on the mesa was selectively etched with $\mathrm{Al}$ etchant Transene $\mathrm{D}$ to produce the $\mathrm{Al}$ strip. A $25 \mathrm{~nm}$-thick layer of insulating $\mathrm{HfO}_{2}$ was grown by atomic layer deposition at a temperature of $90^{\circ} \mathrm{C}$ over the entire sample. Top gates of $\mathrm{Ti} / \mathrm{Au}(5 / 25 \mathrm{~nm})$ were then evaporated and connected to bonding pads with leads of $\mathrm{Ti} / \mathrm{Au}$ $(5 / 250 \mathrm{~nm})$.

Measurements. Electrical measurements were performed by standard lock-in techniques at $166 \mathrm{~Hz}$ by applying the sum of a variable dc bias voltage $V_{\text {sd }}$ and an ac excitation voltage of $3-10 \mu \mathrm{V}$ applied to one of the top ohmic contacts as shown in Fig. 1a. The resulting current across the device was recorded by grounding a bottom ohmic via a low-impedance current-to-voltage converter, and the four terminal voltage was measured by an ac voltage amplifier with an input impedance of $500 \mathrm{M} \Omega$. All measurements were taken in a dilution refrigerator with a base temperature of $20 \mathrm{mK}$ and an electron temperature of $40 \mathrm{mK}$ estimated by the temperature dependence saturation of ZBP conductance ${ }^{3}$.

Data analysis. To highlight the oscillating components of the differential conductance $G\left(B_{\perp}\right)$, a smooth background was subtracted with a low-degree polynomial Savitzky-Golay filter resulting in $\Delta G^{33}$. Analysis of the oscillations was performed by first performing a fast Fourier transform $F(f)$ of $\Delta G\left(B_{\perp}\right)$ using a Hanning window then calculating the power spectral density $P S(f)=|F(f)|^{2}$. The oscillation amplitude $\langle\tilde{A}\rangle$ was obtained by averaging integrated power spectra. The integration was limited to a band in frequency between $f_{1}=0.55 \mathrm{mT}^{-1}$ and $f_{2}=$ $0.75 \mathrm{mT}^{-1}$, spanning the range of a single flux quantum $\Phi_{0}=h / e$ through the area $A$ defined by either the central gate $\left(A_{1}=2.25 \mu \mathrm{m}^{2}\right)$ or the exterior gates $\left(A_{2}=\right.$ $3.1 \mu \mathrm{m}^{2}$ ), where $f=A / \Phi_{0}$ (see Fig. $2 \mathrm{i}-\mathrm{l}$ ).

\section{Data availability}

The data that support the findings of this study are available from the corresponding authors on reasonable request.

Received: 9 March 2020; Accepted: 17 May 2020;

Published online: 25 June 2020

\section{References}

1. Mourik, V. et al. Signatures of Majorana fermions in hybrid superconductor-semiconductor nanowire devices. Science 336, 1003-1007 (2012).

2. Deng, M. et al. Majorana bound state in a coupled quantum-dot hybridnanowire system. Science 354, 1557-1562 (2016).

3. Nichele, F. et al. Scaling of Majorana zero-bias conductance peaks. Phys. Rev. Lett. 119, 136803 (2017).

4. Zhang, H. et al. Quantized Majorana conductance. Nature 556, 74-79 (2018).

5. Albrecht, S. M. et al. Exponential protection of zero modes in Majorana islands. Nature 531, 206-209 (2016) 
6. O'Farrell, E. C. T. et al. Hybridization of sub-gap states in one-dimensional superconductor/semiconductor Coulomb islands. Phys. Rev. Lett. 121, 256803 (2018).

7. Chiu, C.-K., Sau, J. D. \& Sarma, S. D. Conductance of a superconducting Coulomb-blockaded Majorana nanowire. Phys. Rev. B 96, 054504 (2017).

8. Moore, C., Stanescu, T. D. \& Tewari, S. Two-terminal charge tunneling: disentangling Majorana zero modes from partially separated Andreev bound states in semiconductor-superconductor heterostructures. Phys. Rev. B 97, 165302 (2018).

9. Prada, E. et al. From Andreev to Majorana bound states in hybrid superconductor-semiconductor nanowires. Preprint at arXiv:1911.04512 (2019).

10. Deng, M.-T. et al. Nonlocality of Majorana modes in hybrid nanowires. Phys. Rev. B 98, 085125 (2018)

11. Fu, L. Electron teleportation via Majorana bound states in a mesoscopic superconductor. Phys. Rev. Lett. 104, 056402 (2010).

12. Sau, J. D., Swingle, B. \& Tewari, S. Proposal to probe quantum nonlocality of Majorana fermions in tunneling experiments. Phys. Rev. B 92, 020511 (2015).

13. Vijay, S. \& Fu, L. Teleportation-based quantum information processing with Majorana zero modes. Phys. Rev. B 94, 235446 (2016).

14. Hell, M., Flensberg, K. \& Leijnse, M. Distinguishing Majorana bound states from localized Andreev bound states by interferometry. Phys. Rev. B 97, 161401 (2018).

15. Drukier, C., Zirnstein, H.-G., Rosenow, B., Stern, A. \& Oreg, Y. Evolution of the transmission phase through a Coulomb-blockaded Majorana wire. Phys. Rev. B 98, 161401 (2018).

16. Aharonov, Y. \& Bohm, D. Significance of electromagnetic potentials in the quantum theory. Phys. Rev. 115, 485 (1959).

17. Yacoby, A., Schuster, R. \& Heiblum, M. Phase rigidity and $h / 2 e$ oscillations in a single-ring Aharonov-Bohm experiment. Phys. Rev. B 53, 9583 (1996).

18. Schuster, R. et al. Phase measurement in a quantum dot via a double-slit interference experiment. Nature 385, 417-420 (1997).

19. Avinun-Kalish, M., Heiblum, M., Zarchin, O., Mahalu, D. \& Umansky, V. Crossover from 'mesoscopic' to 'universal' phase for electron transmission in quantum dots. Nature 436, 529-533 (2005).

20. Edlbauer, H. et al. Non-universal transmission phase behaviour of a large quantum dot. Nat. Commun. 8, 1710 (2017).

21. Shabani, J. et al. Two-dimensional epitaxial superconductor-semiconductor heterostructures: a platform for topological superconducting networks. Phys. Rev. B 93, 155402 (2016).

22. Hekking, F. W. J., Glazman, L. I., Matveev, K. A. \& Shekhter, R. I. Coulomb blockade of two-electron tunneling. Phys. Rev. Lett. 70, 4138 (1993).

23. Van Heck, B., Lutchyn, R. M. \& Glazman, L. I. Conductance of a proximitized nanowire in the Coulomb blockade regime. Phys. Rev. B 93, 235431 (2016).

24. Tuominen, M. T., Hergenrother, J. M., Tighe, T. S. \& Tinkham, M. Experimental evidence for parity-based 2e periodicity in a superconducting single-electron tunneling transistor. Phys. Rev. Lett. 69, 1997 (1992).

25. Eiles, T. M., Martinis, J. M. \& Devoret, M. H. Even-odd asymmetry of a superconductor revealed by the Coulomb blockade of Andreev reflection. Phys. Rev. Lett. 70, 1862 (1993).

26. Higginbotham, A. P. et al. Parity lifetime of bound states in a proximitized semiconductor nanowire. Nat. Phys. 11, 1017-1021 (2015).

27. Shen, J. et al. Parity transitions in the superconducting ground state of hybrid InSb-Al Coulomb islands. Nat. Commun. 9, 4801 (2018).

28. Stanescu, T. D., Lutchyn, R. M. \& Sarma, S. D. Dimensional crossover in spin-orbit-coupled semiconductor nanowires with induced superconducting pairing. Phys. Rev. B 87, 094518 (2013).

29. Karzig, T. et al. Scalable designs for quasiparticle-poisoning-protected topological quantum computation with Majorana zero modes. Phys. Rev. B 95, 235305 (2017)
30. Plugge, S., Rasmussen, A., Egger, R. \& Flensberg, K. Majorana box qubits. New J. Phys. 19, 012001 (2017)

31. Osca, J., Ruiz, D. \& Serra, L. Effects of tilting the magnetic field in onedimensional Majorana nanowires. Phys. Rev. B 89, 245405 (2014).

32. Tinkham, M. Introduction to Superconductivity (Courier Corporation, 2004)

33. Savitzky, A. \& Golay, M. J. Smoothing and differentiation of data by simplified least squares procedures. Anal. Chem. 36, 1627-1639 (1964).

\section{Acknowledgements}

This work was supported by Microsoft Corporation, the Danish National Research Foundation, and the Villum Foundation. F.N. acknowledges support from European Research Commission, Grant No. 804273. We thank Karsten Flensberg, Joshua Folk, Michael Hell, Andrew Higginbotham, Torsten Karzig, Panagiotis Kotetes, Martin Leijnse, Tommy Li, Alice Mahoney, and Ady Stern for useful discussions.

\section{Author contributions}

T.W., C.T., S.G., R.K., G.C.G. and M.J.M. developed and grew the InAs/Al heterostructure. A.M.W. and A.F. designed and fabricated the devices. A.M.W. and A.F. performed the measurements. Data analysis was done by A.M.W. and A.F with input from C.M.M. and F.N. C.M.M. and F.N. conceived the experiment. A.M.W. and A.F. interpreted the data with input from E.C.T.O., A.C.C.D., C.M.M. and F.N. The manuscript was written by A.M.W., A.F., C.M.M. and F.N. with suggestions from all other authors.

\section{Competing Interests}

The authors declare no competing interests.

\section{Additional information}

Supplementary information is available for this paper at https://doi.org/10.1038/s41467 020-16988-x.

Correspondence and requests for materials should be addressed to C.M.M. or F.N.

Peer review information Nature Communications thanks Ramón Aguado and the other, anonymous, reviewer(s) for their contribution to the peer review of this work

Reprints and permission information is available at http://www.nature.com/reprints

Publisher's note Springer Nature remains neutral with regard to jurisdictional claims in published maps and institutional affiliations.

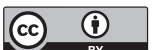

Open Access This article is licensed under a Creative Common Attribution 4.0 International License, which permits use, sharing, adaptation, distribution and reproduction in any medium or format, as long as you give appropriate credit to the original author(s) and the source, provide a link to the Creative Commons license, and indicate if changes were made. The images or other third party material in this article are included in the article's Creative Commons license, unless indicated otherwise in a credit line to the material. If material is not included in the article's Creative Commons license and your intended use is not permitted by statutory regulation or exceeds the permitted use, you will need to obtain permission directly from the copyright holder. To view a copy of this license, visit http://creativecommons.org/ licenses/by/4.0/

(c) The Author(s) 2020 